\title{
Transverse space-charge induced microbunching instability in high-brightness electron bunches
}

\author{
M. Venturini* and J. Qiang \\ Lawrence Berkeley National Laboratory, Berkeley, California 94720-8211, USA
}

(Received 5 March 2015; published 11 May 2015)

\begin{abstract}
We identify a new mechanism for generation of the microbunching instability that involves the transverse rather than the longitudinal component of the self-fields. We introduce an analytical model that captures the essential features of the effect and use the model to interpret results from recent LCLS-II design studies.
\end{abstract}

DOI: 10.1103/PhysRevSTAB.18.054401

PACS numbers: 29.27.-a, 41.60.Cr, 41.75.Fr

\section{INTRODUCTION}

The microbunching instability is a pervasive occurrence in accelerator drivers of $\mathrm{x}$-ray free-electron lasers (FELs) with potentially adverse effects on machine operation and performance. First anticipated in simulations [1] and analytical models [2-5], it has later found extensive experimental confirmation [6-13]. In its common manifestation the instability is driven by the longitudinal component of the beam self-fields originating from coherent synchrotron radiation (CSR) and/or space charge (with the latter usually being dominant). Irregularities in the beam density (shot noise or noise in the electron beam sources) can seed self-fields on the $\mu \mathrm{m}$ scale, which then modify the energy profile along the bunch. As the beam travels through dispersive beam lines (bunch compressors, distribution lines) the modified energy profile causes differential longitudinal slippage and induces the formation of ripples (microbunching) in the current profile on the same $\mu \mathrm{m}$ scale, further amplifying the self-fields. The net result of this feedback loop can be an unacceptable degradation of the beam brightness.

In this paper we identify a new mechanism for the generation of microbunching in which the driver of the instability is the transverse rather than the longitudinal component of the self-fields. We will refer to this effect as transverse space-charge (TSC) induced microbunching. While the conditions for this effect are less common than those leading to longitudinal space-charge (LSC) induced microbunching (a relatively long dispersive section and small geometric emittance may be necessary) they can occur in practical lattice solutions, as we have learned from our LCLS-II [14] design studies.

For an intuitive understanding, consider a model beam with negligible horizontal width $\left(x_{0}=0\right)$ but finite

\footnotetext{
*MVenturini@lbl.gov

Published by the American Physical Society under the terms of the Creative Commons Attribution 3.0 License. Further distribution of this work must maintain attribution to the author(s) and the published article's title, journal citation, and DOI.
}

angular spread, as schematically represented in Fig. 1(a). Let the beam be uniform in the longitudinal coordinate $z_{0}$ but have a relative energy modulation $\delta=\delta_{p} \cos \left(k z_{0}\right)$. The beam enters a dispersive beam line and a particle horizonal coordinate evolves to $x=R_{12} x_{0}^{\prime}+R_{16} \delta_{p} \cos \left(k z_{0}\right)$, where $R_{12}$ and $R_{16}$ are the transfer matrix entries from the entrance of the beam line to the observation point. The particle experiences longitudinal slippage according to $z=z_{0}+R_{52} x_{0}^{\prime}$, having assumed that $R_{56}$ vanishes or is negligible. Solving the latter equation for $z_{0}$ and inserting the result in the equation for $x$ we obtain $x=R_{12} x_{0}^{\prime}+R_{16} \delta_{p} \cos \left[k\left(z-R_{52} x_{0}^{\prime}\right)\right]$. If we now use this transfer function to map the stripes in Fig. 1(a) we find the pattern shown in Fig. 1(b), indicating the appearance of higher concentration of charge along diagonal ridges on a spacial scale determined by the wave number $k$. We will refer to this pattern as "longitudinal/transverse" (or 2D) microbunching [observed in the $(z, x)$ plane] as distinct from the "longitudinal" microbunching obtained upon projection of the beam density onto the $z$ axis. Observe that the beam at this point has remained longitudinally uniform (no longitudinal microbunching).
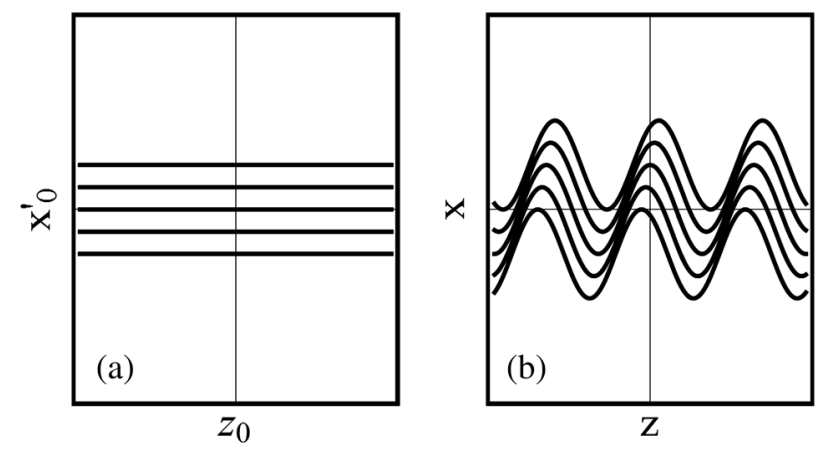

FIG. 1. A beam assumed for simplicity to have vanishing horizontal width $\left(x_{0}=0\right)$ but finite angular spread, uniform density in $z_{0}$, and carrying a small energy modulation, is represented here as a set of five uniform charge lines in the $z_{0} / x_{0}^{\prime}$ plane (left). The beam will exhibit longitudinal/transverse microbunching (right) as it propagates through a dispersive transport line (lumping of charge along diagonal ridges). 
Longitudinal/transverse microbunching will induce a space-charge field with finite transverse component. If strong enough the field will perturb the particle angular coordinate: $x^{\prime} \rightarrow x^{\prime}+\Delta x^{\prime}$, with a kick $\Delta x^{\prime}=\Delta x^{\prime}(z, x)$ exhibiting a similar $(z, x)$ dependance as the charge density. Further transport to the exit of the dispersive line will then cause a slippage $\Delta z=R_{52} \Delta x^{\prime}(z, x)$. This does not demonstrate but it does suggest the appearance of a sinusoidal slippage modulation $\Delta z$, after integrating over the transverse coordinate, i.e., the appearance of longitudinal microbunching. Indeed, we will show that this can be the case in the more refined model discussed in Sec. II. Notice that 2D microbunching disappears once the beam exits the (achromatic) dispersive line. We evaluate the effect of the transverse component of the space-charge self-fields associated with microbunching in the static approximation, widely used in analytical and numerical simulation models, thus neglecting the possible contribution from transients effects.

The possible appearance of 2D microbunching as a result of an energy modulation was noted before [15]. What is new here is the observation that the transverse self-fields induced by this microstructure can generate permanent longitudinal microbunching. Another notable difference is in the origin of the 2D microbunching. In [15], this is seeded by a laser/beam interaction; in our case, it results from an energy modulation induced by upstream microbunching instability. In Sec. III we will use our analytical model to interpret results from macroparticle simulations modeling the LCLS-II lattice.

\section{ANALYTICAL BEAM MODEL FOR LONGITUDINAL/TRANSVERSE MICROBUNCHING}

\section{A. Longitudinal/transverse microbunching within achromat}

We consider a model of longitudinally uniform beam with $n_{z}=N / L_{b}$ line density, Gaussian distribution in the transverse and energy coordinates, and a small sinusoidal energy modulation:

$$
f\left(\boldsymbol{x}_{0}, s_{0}\right)=\frac{n_{z}}{\sqrt{2 \pi} \sigma_{\delta}} e^{-\left(\delta_{0}+\delta_{p} \cos k z_{0}\right)^{2} / 2 \sigma_{\delta}^{2}} f_{x} f_{y},
$$

where $f_{x}=\left(2 \pi \varepsilon_{x 0}\right)^{-1} \exp \left[-\left(\gamma_{x 0} x_{0}^{2}+2 \alpha_{x 0} x_{0} x_{0}^{\prime}+\beta_{x 0} x_{0}^{\prime 2}\right) /\right.$ $\left.2 \varepsilon_{x 0}\right]$ and $x_{0}$ is a particle $6 \mathrm{D}$ coordinate vector. Notice that in the following we will distinguish between the "intrinsic" or invariant $\mathrm{rms}$ horizontal emittance $\varepsilon_{x 0}$, as introduced above, and the projected emittance $\varepsilon_{x}$ in the presence of dispersion. Here and in the following the subscript " 0 " refers to quantities defined at the entrance of the beam line. For $f_{y}$ we assume a similar form, but the details of the vertical motion are mostly irrelevant here.
We do a first-order approximation in the amplitude $\delta_{p}$ of the energy modulation and consider

$$
f\left(\boldsymbol{x}_{0}, s_{0}\right) \simeq f^{(0)}\left(\boldsymbol{x}_{0}, s_{0}\right)+f^{(1)}\left(\boldsymbol{x}_{0}, s_{0}\right)
$$

with $f^{(1)}=\delta_{p} \frac{\partial f\left(\delta_{p}=0\right)}{\partial \delta_{p}}$. The beam is transported along a dispersive beam line, with unperturbed (no space charge) motion described in linear approximation, $\boldsymbol{x}_{0} \rightarrow \boldsymbol{x}=$ $\boldsymbol{R}\left(s_{0} \rightarrow s\right) \boldsymbol{x}_{0}$. For the transfer matrix $\boldsymbol{R}\left(s_{0} \rightarrow s\right)$ we will use the compact notation $\boldsymbol{R}=\boldsymbol{R}(s)$ and following standard convention we write $\eta_{x}=R_{16}, \eta_{x}^{\prime}=R_{26}$ for the dispersion function and derivative. The beam line is an isochronous achromat, $R_{56}\left(s_{f}\right)=\eta_{x}\left(s_{f}\right)=\eta_{x}^{\prime}\left(s_{f}\right)=0$, with bending occurring only in the horizontal plane.

Our first task is to find the beam density in the $z / x$ plane at any point $s_{0} \leq s \leq s_{f}$ within the beam line. If $R_{56}(s) \neq 0$ only over distances that are negligible compared to the overall achromat length, we make a small error if we assume, as we do, that $R_{56}(s)=0$ throughout; this will be the case in the example discussed in Sec. III.

Upon propagation, $f^{(1)}\left(\boldsymbol{x}_{0}, s_{0}\right) \rightarrow f^{(1)}(\boldsymbol{x}, s)=f^{(1)}\left(\boldsymbol{R}^{-1} \boldsymbol{x}\right.$, $s_{0}$ ), and integration over $\delta$, the first-order perturbation to the beam density as observed at location $s$ can be cast in the form

$$
\begin{aligned}
\int d & \delta f^{(1)}(\boldsymbol{x}, s) \\
= & -\delta_{p} n_{z} \frac{\xi \beta_{x} x^{\prime}+\tau \alpha_{x} x}{2 \pi} \frac{\varepsilon_{x 0}}{\varepsilon_{x}^{3}} f_{y} \\
& \times e^{-\left(\tilde{\gamma}_{x} x^{2}+2 \tilde{\alpha}_{x} x x^{\prime}+\tilde{\beta}_{x} x^{\prime 2}\right) / 2 \varepsilon_{x}} \cos \left[k\left(\eta_{x}^{\prime} x-\eta_{x} x^{\prime}+z\right)\right],
\end{aligned}
$$

where we have made use of $R_{51}^{-1}=\eta_{x}^{\prime}, R_{52}^{-1}=-\eta_{x}$ [16], and introduced the projected horizontal rms emittance $\varepsilon_{x}^{2}=\varepsilon_{x 0}^{2}+\varepsilon_{x 0} \mathcal{H} \sigma_{\delta}^{2}$, with $\mathcal{H}=\gamma_{x} \eta_{x}^{2}+2 \alpha_{x} \eta_{x} \eta_{x}^{\prime}+\beta_{x} \eta_{x}^{\prime 2}$ being the dispersion invariant. We also defined the functions

$$
\begin{aligned}
& \xi=\alpha_{x} \eta_{x} / \beta_{x}+\eta_{x}^{\prime}, \\
& \tau=\gamma_{x} \eta_{x} / \alpha_{x}+\eta_{x}^{\prime},
\end{aligned}
$$

and the effective Twiss parameters $\tilde{\beta}_{x}=\left(\beta_{x} \varepsilon_{x 0}+\eta_{x}^{2} \sigma_{\delta}^{2}\right) / \varepsilon_{x}$, $\tilde{\gamma}_{x}=\left(\gamma_{x} \varepsilon_{x 0}+\eta_{x}^{\prime 2} \sigma_{\delta}^{2}\right) / \varepsilon_{x}$, and $\tilde{\alpha}_{x}=\left(\alpha_{x} \varepsilon_{x 0}-\eta_{x} \eta_{x}^{\prime} \sigma_{\delta}^{2}\right) / \varepsilon_{x}$. Notice that $\sigma_{x}^{2}=\varepsilon_{x} \tilde{\beta}_{x}$ is the horizontal rms width including the enlargement due to dispersion.

After integration, $\rho^{(1)}(z, x, y ; s)=\int d \delta d x^{\prime} d y^{\prime} f^{(1)}(\boldsymbol{x}, s)$, we find

$$
\begin{aligned}
\rho^{(1)}= & -\delta_{p} n_{3 d} e^{-x^{2} / 2 \sigma_{x}^{2}-y^{2} / 2 \sigma_{y}^{2}} e^{-\varepsilon_{x} \eta_{x}^{2} k^{2} / 2 \tilde{\beta}_{x}} \\
& \times \eta_{x}\left[\frac{x}{\sigma_{x}^{2}} \cos (k \tilde{\xi} x+k z)+\frac{\beta_{x} \varepsilon_{x 0}}{\sigma_{x}^{2}} k \xi \sin (k \tilde{\xi} x+k z)\right],
\end{aligned}
$$


where $n_{3 d}=n_{z} / 2 \pi \sigma_{x} \sigma_{y}$ and

$$
\tilde{\xi}=\tilde{\alpha}_{x} \eta_{x} / \tilde{\beta}_{x}+\eta_{x}^{\prime}
$$

\section{B. Transverse self-field and kick}

We will consider the case, relevant for the example discussed in Sec. III, where the sin term in (5) is dominant $\left(k \xi \gg 1 / \sigma_{x}\right)$ and solve the Poisson equation in the beam comoving frame (subscript " $b$ "):

$$
\nabla^{2} \phi_{b}^{(1)}=\frac{e \rho_{b}^{(1)}}{\epsilon_{0}},
$$

with

$$
\rho_{b}^{(1)} \simeq \frac{\hat{\rho}^{(1)}}{\gamma} \sin \left(k \tilde{\xi} x_{b}+k z_{b} / \gamma\right),
$$

and

$$
\hat{\rho}^{(1)}=-\delta_{p} n_{3 d} \eta_{x} \frac{\beta_{x} \varepsilon_{x 0}}{\sigma_{x}^{2}} k \xi e^{-\varepsilon_{x} \eta_{x}^{2} k^{2} / 2 \tilde{\beta}_{x}} .
$$

We have neglected the slow-varying dependence of $\rho^{(1)}$ with respect to $x, y$, amounting to setting $x=y=0$ in the exponential terms in (5), and by the same token, we neglect the contribution to the dynamics from the zero-order component of $\phi_{b}$. The solution to the Poisson equation is

$$
\phi_{b}^{(1)}\left(z_{b}, x_{b} ; s\right)=-\frac{e \hat{\rho}^{(1)}}{\gamma \epsilon_{0}} \frac{\sin \left(k \tilde{\xi} x_{b}+k z_{b} / \gamma\right)}{k^{2}\left(\gamma^{-2}+\tilde{\xi}^{2}\right)},
$$

yielding the horizontal component of the electric field $E_{b, x}=-\partial_{x} \phi_{b}^{(1)}$ :

$$
E_{b, x}=\frac{e \hat{\rho}^{(1)} \tilde{\xi}}{\gamma \epsilon_{0}} \frac{\cos \left(k \tilde{\xi} x_{b}+k z_{b} / \gamma\right)}{k\left(\gamma^{-2}+\tilde{\xi}^{2}\right)} .
$$

Upon transformation to the lab frame $E_{x}=\gamma E_{b, x}, B_{y} \simeq$ $c \gamma E_{b, x}$ for the net transverse collective force we find $F_{x}=-e\left(E_{x}-c B_{y}\right)=-e \gamma E_{b, x} / \gamma^{2}$, and therefore

$$
\frac{d x^{\prime}}{d s}=\frac{F_{x}}{c p_{0}}=-\frac{4 \pi r_{c} \hat{\rho}^{(1)} \tilde{\xi}}{\gamma k\left(1+\gamma^{2} \tilde{\xi}^{2}\right)} \cos (k \tilde{\xi} x+k z),
$$

now expressed in the lab coordinates and valid in the ultrarelativistic approximation; $r_{c}$ is the electron classical radius.

\section{Longitudinal microbunching at exit of achromat}

As a consequence of the TSC-induced transverse kick the beam distribution through first order in $\delta_{p}$ evolves according to

$$
f\left(\boldsymbol{x}_{f}, s_{f}\right)=f^{(0)}\left(\boldsymbol{x}_{0}, s_{0}\right)+\int_{s_{0}}^{s_{f}} d s \frac{\partial f^{(0)}(\boldsymbol{x}, s)}{\partial x^{\prime}} \frac{d x^{\prime}}{d s},
$$

with $d x^{\prime} / d s$ given by (12). Both sides of the above equation are understood to be functions of the dynamical variables $\boldsymbol{x}_{f}$ at $s_{f}$, e.g., $\boldsymbol{x}_{0}=\boldsymbol{R}^{-1}\left(s_{0} \rightarrow s_{f}\right) \boldsymbol{x}_{f}$. To avoid notation clutter in the following we omit indicating the $s$ dependence in the distribution function.

The goal here is to calculate the bunching as seen at the exit of the beam line [17],

$$
b_{k}=\frac{2}{N} \int d^{4} x_{f} \sin \left(k z_{f}\right) f\left(\boldsymbol{x}_{f}\right) .
$$

The sine transform of the first term on the rhs of (13) vanishes (no bunching in the absence of space charge) and we are left with

$$
b_{k}=\frac{2}{N} \int d^{4} \boldsymbol{x}_{f} \sin \left(k z_{f}\right) \int_{s_{0}}^{s_{f}} d s \frac{\partial f^{(0)}(\boldsymbol{x})}{\partial x^{\prime}} \frac{d x^{\prime}}{d s} .
$$

We perform a change of integration variables $\boldsymbol{x}_{f} \rightarrow \boldsymbol{x}=\boldsymbol{R}^{-1}\left(s \rightarrow s_{f}\right) \boldsymbol{x}_{f}$,

$$
b_{k}=\frac{2}{N} \int_{s_{0}}^{s_{f}} d s \int d^{4} \boldsymbol{x} \sin \left[k z_{f}(\boldsymbol{x})\right] \frac{\partial f^{(0)}(\boldsymbol{x})}{\partial x^{\prime}} \frac{d x^{\prime}}{d s},
$$

integrate by parts

$$
b_{k}=-\frac{2 k}{N} \int_{s_{0}}^{s_{f}} d s \int d^{4} \boldsymbol{x} \frac{\partial z_{f}(\boldsymbol{x})}{\partial x^{\prime}} \cos \left[k z_{f}(\boldsymbol{x})\right] f^{(0)}(\boldsymbol{x}) \frac{d x^{\prime}}{d s},
$$

and recognize that $\frac{\partial z_{f}(\boldsymbol{x})}{\partial x^{\prime}}=R_{52}\left(s \rightarrow s_{f}\right)=-\eta_{x}(s)$. Upon a further variable transformation $\boldsymbol{x} \rightarrow \boldsymbol{x}_{0}=\boldsymbol{R}^{-1}\left(s_{0} \rightarrow s\right) \boldsymbol{x}$ we can write the above integral as

$$
b_{k}=\frac{2 k}{N} \int_{s_{0}}^{s_{f}} d s \eta_{x} \int d^{4} \boldsymbol{x}_{0} \cos \left(k z_{0}\right) f^{(0)}\left(\boldsymbol{x}_{0}\right) \frac{d x^{\prime}}{d s},
$$

having observed that $z_{f}\left(\boldsymbol{x}_{0}\right)=z_{0}$ (isochronous transport line).

We write the expression for the transverse space-charge kick (12) as

$$
\begin{aligned}
\frac{d x^{\prime}}{d s} & =A \cos \left[k \tilde{\xi} x\left(\boldsymbol{x}_{0}\right)+k z\left(\boldsymbol{x}_{0}\right)\right] \\
& =A \cos \left[k\left(x_{0} r_{x}+x_{0}^{\prime} r_{x^{\prime}}+\tilde{\xi} \eta_{x}\right)+k z_{0}\right]
\end{aligned}
$$

with $r_{x}=\tilde{\xi} R_{11}+R_{51}, r_{x^{\prime}}=\tilde{\xi} R_{12}+R_{52}$ and $\tilde{\xi}$ defined in (6), and $A(s)=-\frac{4 \pi r_{c} \hat{\rho}^{(1)} \tilde{\xi}}{\gamma k\left(1+\gamma^{2} \tilde{\xi}^{2}\right)}$. The integration in Eq. (17) involves the evaluation of 


$$
\begin{aligned}
& \int d x_{0} d x_{0}^{\prime} \frac{e^{-\frac{x_{0}^{2}+\left(\alpha_{x} x_{x}+\beta_{x} x_{0}^{\prime}\right)^{2}}{2 \varepsilon_{x 0} \beta_{x}}}}{2 \pi \varepsilon_{x 0}} \cos \left[k\left(x_{0} r_{x}+x_{0}^{\prime} r_{x^{\prime}}\right)\right] \\
& =e^{-\varepsilon_{x 0} k^{2}\left(r_{x}^{2} \beta_{x 0}-2 r_{x} r_{x^{\prime}} \alpha_{x 0}+\gamma_{x 0}^{2} r_{x^{\prime}}^{2}\right) / 2} \\
& =\exp \left[-\frac{\varepsilon_{x 0} \eta_{x}^{2} k_{x}^{2}}{\tilde{\beta}_{x}}\left(\zeta-\frac{\beta_{x}}{2 \tilde{\beta}_{x}}\right)\right]
\end{aligned}
$$

where in the last expression we introduced the "mismatch" lattice function

$$
\zeta=\frac{1}{2}\left(\tilde{\gamma}_{x} \beta_{x}-2 \tilde{\alpha}_{x} \alpha_{x}+\tilde{\beta}_{x} \gamma_{x}\right),
$$

and we made use of the relationships $R_{51}=\eta_{x} R_{21}-\eta_{x}^{\prime} R_{11}$ and $R_{52}=\eta_{x} R_{22}-\eta_{x}^{\prime} R_{12}$ to write $r_{x}=\eta_{x}\left(\tilde{\alpha}_{x} R_{11} / \tilde{\beta}_{x}+\right.$ $\left.R_{21}\right)$ and $r_{x^{\prime}}=\eta_{x}\left(\tilde{\alpha}_{x} R_{12} / \tilde{\beta}_{x}+R_{22}\right)$.

The final result reads

$$
\begin{aligned}
b_{k}= & \delta_{p} \frac{2 I k}{\gamma I_{A}} \int_{s_{0}}^{s_{f}} d s \frac{\eta_{x}^{2}}{\sigma_{x} \sigma_{y}} \frac{\beta_{x} \varepsilon_{x 0}}{\sigma_{x}^{2}} \frac{\tilde{\xi}}{1+\gamma^{2} \tilde{\xi}^{2}} \\
& \times \exp \left[-\frac{\varepsilon_{x 0} \eta_{x}^{2} k^{2}}{\tilde{\beta}_{x}}\left(\tilde{\zeta}+\frac{1}{2} \frac{\varepsilon_{x}}{\varepsilon_{x 0}}-\frac{\beta_{x}}{2 \tilde{\beta}_{x}}\right)\right] e^{-\left(k \tilde{\xi} \eta_{x} \sigma_{\delta}\right)^{2} / 2} .
\end{aligned}
$$

In the limit of vanishing uncorrelated energy spread $\sigma_{\delta} \rightarrow 0$ we have $\tilde{\alpha}_{x} \rightarrow \alpha_{x}, \tilde{\beta}_{x} \rightarrow \beta_{x}, \tilde{\gamma}_{x} \rightarrow \gamma_{x}, \zeta \rightarrow 1$, and $\varepsilon_{x} \rightarrow \varepsilon_{x 0}$, and Eq. (21) reduces to

$$
b_{k}=\delta_{p} \frac{2 I k}{\gamma I_{A}} \int_{s_{0}}^{s_{f}} d s \frac{\eta_{x}^{2}}{\sigma_{x} \sigma_{y}} \frac{\xi^{2}}{1+\gamma^{2} \xi^{2}} e^{-\frac{\varepsilon_{x} \eta_{0}^{2} k_{x}^{2}}{\beta_{x}}} .
$$

A further simplification can be made if $\xi_{\gamma} \gg 1$,

$$
b_{k}=\delta_{p} \frac{2 I k}{\varepsilon_{x n} \gamma^{2} I_{A}} \int_{s_{0}}^{s_{f}} d s \frac{\eta_{x}^{2}}{\sqrt{\beta_{x} \beta_{y}}} e^{-\frac{\varepsilon_{x} \eta_{\gamma}^{2} k^{2}}{\gamma \beta_{x}}},
$$

having expressed the result in terms of the normalized emittance $\varepsilon_{x n}=\gamma \varepsilon_{x 0}$.

\section{EXAMPLE FROM LCLS-II DESIGN STUDIES}

As an illustration of TSC-induced longitudinal microbunching we present an example from our studies of LCLSII, an early-stage project hosted at SLAC for a new high rep-rate FEL-based light source. To conform to existing facilities the superconducting linac will be about $2 \mathrm{~km}$ away from the undulator hall. The long transport beam line of the fully accelerated $4 \mathrm{GeV}$ beam will include several dispersive sections. Here we consider the first of these (DL1), as presently under consideration, Fig. 2. Placed at about $700 \mathrm{~m}$ downstream of the second bunch-compressor chicane and $300 \mathrm{~m}$ from the end of the linac, it is about $80 \mathrm{~m}$ long and consists of a pair of $1 \mathrm{~m}$ long dipoles in a dogleg configuration separated by a FODO channel with $2 \pi$ phase advance to cancel dispersion. In the actual design the dipoles are rotated by about $60 \mathrm{deg}$ to move the beam to the top of the tunnel and bypass a portion of the existing copper machine. We study a variant of the dogleg design where the dipoles are not rotated and bending only occurs in the horizontal plane. The effect of interest is unaffected by this change.

The dogleg is complemented by two small chicanes (each contributing about $R_{56} \simeq-100 \mu \mathrm{m}$ ) placed right upstream of the first and downstream of the second bend (each dogleg dipole contributing about $R_{56} \simeq 100 \mu \mathrm{m}$ ) and intended to provide local $R_{56}$ cancellation [19]. In earlier studies these were found to be effective at mitigating LSCinduced microbunching. However, somewhat surprisingly the $R_{56}$ compensation did not completely suppress microbunching amplification. Some of the residual gain was found to be contributed by the nonlinear term $T_{566}$ of the dogleg momentum compaction in combination with nonnegligible energy chirp (the energy chirp is not fully removed from the beam until it enters the FEL). The remaining contribution to the observed microbunching was unequivocally found to be due to TSC by comparing macroparticle simulations with and without account of the effect of TSC on the particle dynamics.

The simulation performed for this study was carried out with the code IMPACT [20], tracking an idealized ("waterbag" [21]) beam distribution having the same characteristics as those of a nominal $100 \mathrm{pC}$ bunch from the injector. The simulations include the effect of the laser heater and CSR, but the latter has a negligible role on the effect of interest here, and a number of macroparticles equal to that of physical electrons. In the simulation the beam energy chirp was removed before DL1 to exclude $T_{566}$ effects.

The microbunching amplification observed through DL1 is due to the presence of an energy modulation on the beam,

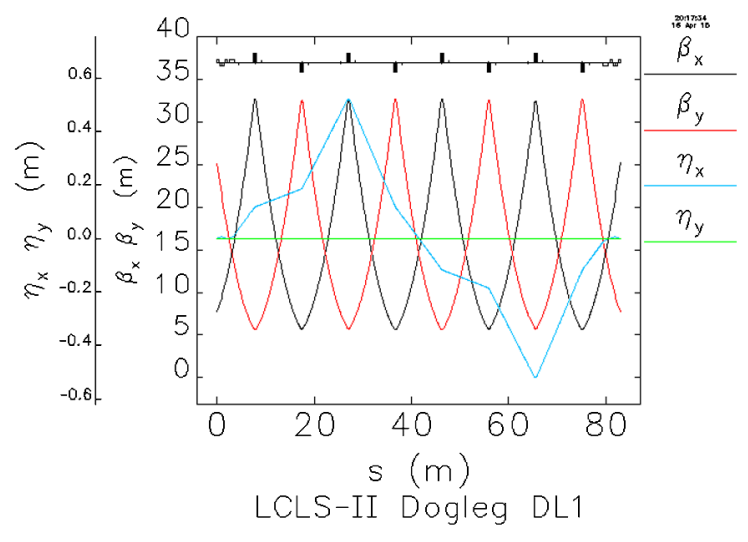

FIG. 2. Lattice functions (evaluated with ELEGANT [18]) along the achromatic dogleg DL1, presently under consideration for LCLS-II and used in our example illustrating TSC-induced longitudinal microbunching. At both extremities of the beam line two small four-bend chicanes provide local compensation of the linear momentum compaction $R_{56}$. 


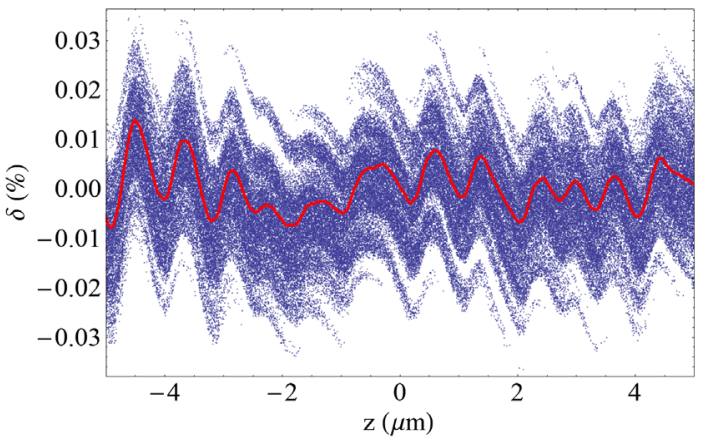

FIG. 3. Longitudinal phase space of the beam core at the entrance of DL1. The red curve is the slice centroid energy. The apparent $\sim 1 \mu \mathrm{m}$ energy modulation is the result of LSC during acceleration and transport following the second bunch compressor, placed about $700 \mathrm{~m}$ upstream of DL1.

see Fig. 3. This modulation has its origin in an imperfect damping of the microbunching instability by the laser heater. When the laser heater is operated at its design settings (equivalent to generating $\sigma_{E}=7 \mathrm{keV}$ rms slice energy spread) a small 2\%-3\% [22] longitudinal microbunching stemming from shot noise appears at the exit of the second bunch compressor with spectrum peaked at about $\lambda \simeq 1 \mu \mathrm{m}$. This is sufficient to induce an energy modulation in a range of values including $\delta_{p} \sim 0.005 \%$ by the time the beam enters the dogleg. As expected from the analysis in the previous section, the energy modulation turns into longitudinal/transverse bunching, see Fig. 4 . The analytical model predicts fairly well the points of largest longitudinal/transverse bunching along the transport line, Fig. 5; however, not unexpectedly, it is less accurate for a quantitative estimate of the bunching amplitude. The better prediction is obtained using Eq. (22) valid for vanishing slice-energy spread $\sigma_{\delta}$ rather than Eq. (21). The latter is valid under the assumption of a Gaussian energy spread distribution, which appears to overestimate the effect of mixing thus predicting smaller bunching. (The slice energy

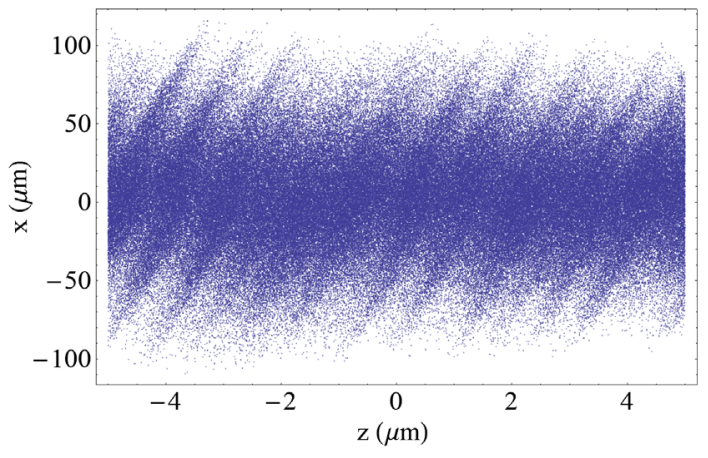

FIG. 4. Section $x / z$ of the beam phase space observed just before the first quad of DL1 showing the longitudinal/transverse microbunching induced by the energy modulation of Fig. 3. The picture is taken where the magnitude of the $2 \mathrm{D}$ microbunching is the largest, see Fig. 5.

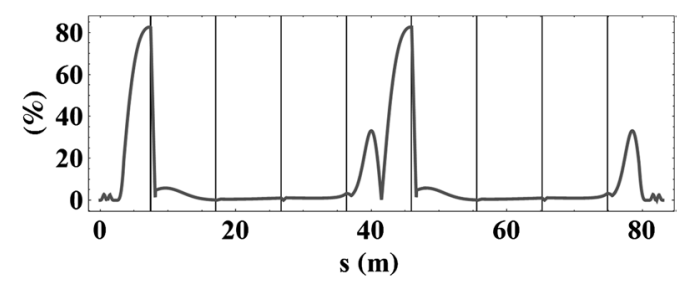

FIG. 5. Quantitative measure of the longitudinal/transverse bunching along the dogleg, defined as the first-order amplitude $\left|\hat{\rho}^{(1)}\right| / n_{3 d}$ as calculated from Eq. (9) and the DL1 lattice functions. The quantity plotted here measures the contrast in the density of the diagonal bands observed e.g., in Fig. 4. The vertical lines mark the location of the quadrupoles. Longitudinal/ transverse bunching requires finite dispersion $\eta_{x}$ but it is exponentially suppressed at points where the dispersion becomes too large $\eta_{x}^{2} \gtrsim \tilde{\beta}_{x} / \varepsilon_{x} k^{2}$.

spread is $\sigma_{\delta} \simeq 0.013 \%$.) Indeed, the energy distribution in the simulated beam, including the effect of the laser heater, has a markedly non-Gaussian profile. For an example of reduced microbunching due to non-Gaussian beam distribution in a different but related context see [23]. Another limitation of the analytical model, of course, is the assumption that one can approximate the microbunching instability induced energy modulation by an effective purely sinusoidal modulation.

Using $\delta_{p}=0.01 \%$ (on the high end of the numerical values observed for the modulation amplitude), $\lambda=2 \pi / k=1 \mu \mathrm{m}, I=850 \mathrm{~A}, \gamma \varepsilon_{x 0}=0.3 \mu \mathrm{m}$ (appropriate for $100 \mathrm{pC}$ bunches), and $E=4 \mathrm{GeV}$ we obtain $b_{k} \simeq 2.5 \%$ for the TSC induced bunching, about consistent with the result from the simulations, see Fig. 6 [24].

This level of bunching would seem relatively modest but is, in fact, significant for LCLS-II, as further amplification of the microbunching takes place in the long transport beam line downstream of DL1. A more detailed discussion of the significance of this and other microbunching instability aspects in LCLS-II will be reported elsewhere.

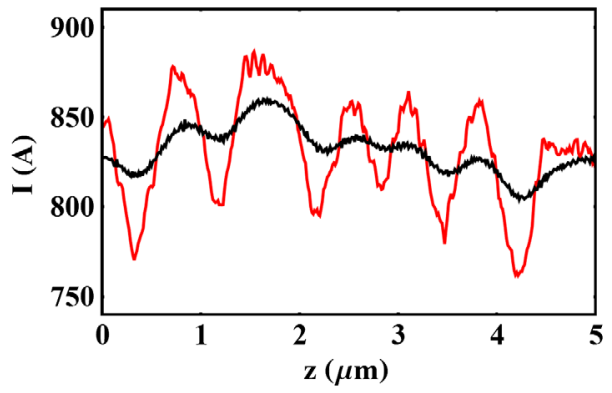

FIG. 6. The beam current profile as seen at the entrance of DL1 (black curve) shows $\sim 1 \%$ longitudinal bunching amplitude at about $\lambda \simeq 1 \mu \mathrm{m}$ wavelength. By the exit of the dogleg (red curve) bunching has grown to about $4 \%$, implying a net $\sim 3 \%$ contribution from TSC in the dogleg. The quoted numbers are about the middle of the observed value ranges. 


\section{CONCLUSIONS}

In this paper we focused our analysis on the effect of the transverse component of the space-charge associated with longitudinal/transverse microbunching. We have developed a simple analytical model that captures the essential beamphysics effects, including prediction of longitudinal bunching amplification from transport through a dogleg beam line, first observed in numerical simulations of the LCLS-II lattice. In spite of several simplifying assumptions, the analytical model retains the capability to predict the magnitude of the effect as determined numerically, while offering insight into the scaling with respect to the basic parameters. In particular, a sensitive parameter is the transverse geometric emittance: it is important for the beam to be sufficiently bright for the 2D microbunching not to be washed out. Finally, for the effect to occur the beam has to accrue an energy modulation with suitable dominant wavelength before the beam reaches the dispersive region of interest (e.g., a dogleg); "conventional" microbunching instability in the upstream linac can generate the necessary modulation, depending on the overall machine design and settings (including those of the laser heater).

The longitudinal component of the field could in principle have measurable consequences on the beam energy spread ("trickle" heating [15]) but our simulation shows this to be a negligible effect for the example of Sec. III.

The DL1 beam line was designed to be locally nearly isochronous by introducing small compensating chicanes intended to minimize the microbunching gain. In light of the present analysis it is possible that partial $R_{56}$ compensation through the dogleg could represent a more effective solution as it would allow TSC- and LSC-induced longitudinal microbunching to weigh against each other. This and the TSC effect on transverse emittance will be addressed in further studies.

\section{ACKNOWLEDGMENTS}

We acknowledge useful discussions with the LCLS-II design team, Mark Woodley for the LCLS-II lattice design, P. Emma, R. Ryne, C. Mitchell, A. Zholents, and M. Zolotorev for valuable comments. This work was supported in part by Department of Energy Contract No. DE-AC0205CH11231 and the LCLS-II Project.

[1] M. D. Borland, Y.-C. Chae, J. W. Lewellen, S. V. Milton, R. Soliday, V. Bharadwaj, P. Emma, P. Krejcik, H.-D. Nuhn, and W. M. Fawley, Nucl. Instrum. Methods Phys. Res., Sect. A 483, 268 (2002).

[2] E. L. Saldin, E. A. Schneidmiller, and M. V. Yurkov, Nucl. Instrum. Methods Phys. Res., Sect. A 483, 516 (2002).

[3] Z. Huang and K.-J. Kim, Phys. Rev. ST Accel. Beams 5, 074401 (2002).
[4] M. Venturini, Phys. Rev. ST Accel. Beams 10, 104401 (2007); 11, 034401 (2008).

[5] A. Marinelli and J. Rosenzweig, Phys. Rev. ST Accel. Beams 13, 110703 (2010).

[6] T. Shaftan and Z. Huang, Phys. Rev. ST Accel. Beams 7, 080702 (2004).

[7] D. Dowell et al., in Proceedings of the 29th Free Electron Laser Conference, Novosibirsk, Russia (BINP, Novosibirsk, 2007).

[8] H. Loos et al., in Proceedings of the 30th International Free Electron Laser Conference (POSTECH, Gyeongju, 2008), p. 485.

[9] A. H. Lumpkin, N. S. Sereno, W. J. Berg, M. Borland, Y. Li, and S. J. Pasky, Phys. Rev. ST Accel. Beams 12, 080702 (2009).

[10] S. Wesch, C. Behrens, B. Schmidt, and P. Schmuser, in Proceedings of the 31st International Free Electron Laser Conference (FEL 09), Liverpool, UK (STFC Daresbury Laboratory, Warrington, 2009), p. 619.

[11] S. Seletskiy, Y. Hidaka, J. B. Murphy, B. Podobedov, H. Qian, Y. Shen, X. J. Wang, and X. Yang, Phys. Rev. ST Accel. Beams 14, 110701 (2011).

[12] S. Di Mitri and S. Spampinati, Phys. Rev. Lett. 112, 134802 (2014).

[13] D. Ratner, C. Behrens, Y. Ding, Z. Huang, A. Marinelli, T. Maxwell, and F. Zhou, Phys. Rev. ST Accel. Beams 18, 030704 (2015).

[14] J. Galayda, in Proceedings of the 5th International Particle Accelerator Conference (HZDR, Dresden, 2014), p. 935.

[15] Z. Huang et al., Phys. Rev. ST Accel. Beams 13, 020703 (2010).

[16] We alert the reader that these and other relationships between matrix entries mentioned later depend on the sign convention for the dynamical coordinates. We adopted the convention that $z<0$ for a particle in the bunch head and, in this section, $\delta=-\left(E-E_{r}\right) / E_{r}$. When reporting on the numerical simulations in Sec. III we revert to the more common choice $\delta=\left(E-E_{r}\right) / E_{r}$. Here $E_{r}$ is the energy of the reference particle on the design orbit.

[17] The cosine transform can be shown to vanish, given our choice of phase for the initial energy modulation.

[18] M. Borland, Argonne National Laboratory Advanced Photon Source Report No. LS-287, 2000.

[19] M. Venturini and A. Zholents, Nucl. Instrum. Methods Phys. Res., Sect. A 593, 53 (2008).

[20] J. Qiang, R. D. Ryne, M. Venturini, A. A. Zholents, and I. V. Pogorelov, Phys. Rev. ST Accel. Beams 12, 100702 (2009).

[21] Randomly populated hyperellipsoid in the 6D phase space with uniform averaged density.

[22] This bunching is essentially the same as that observed at the entrance of DL1, black curve in Fig. 6; its magnitude decreases slightly as the beam travels toward DL1 because of slow but non-negligible plasma oscillation.

[23] E. Ferrari, E. Allaria, W. Fawley, L. Giannessi, Z. Huang, G. Penco, and S. Spampinati, Phys. Rev. Lett. 112, 114802 (2014).

[24] Unfortunately, the phases of the longitudinal bunching upstream of the dogleg causing the energy modulation and that of the longitudinal bunching induced by TSC are the same and the two amplitudes add up. 